\title{
Stress relaxation behavior of organically modified montmorillonite filled natural rubber/nitrile rubber nanocomposites
}

\author{
Hanna J. Maria a,f ${ }^{\text {a }}$ Nathalie Lyczko ${ }^{\mathrm{e}}$, Ange Nzihou ${ }^{\mathrm{e}}$, Kuruvilla Joseph ${ }^{\mathrm{d}}$, Cherian Mathew ${ }^{\mathrm{f}}$, Sabu Thomas ${ }^{\mathrm{a}, \mathrm{b}, \mathrm{c}, \mathrm{g}, *}$
}

\author{
a School of Chemical Sciences, Mahatma Gandhi University, Priyadarshini Hills, Kottayam, Kerala, India \\ ${ }^{\mathrm{b}}$ Center of Excellence for Polymer Materials and Technologies, Tehnoloski park 24, SI 1000 Ljubljana, Slovenia \\ ${ }^{c}$ Universiti of Teknologi MARA, 40450, Shah Alam, Selangor, Malaysia \\ d Indian Institute of Space Science and Technology, Dept of Chemistry, Thiruvanathapuram, Kerala, India \\ e Université de Toulouse, Mines Albi, CNRS, Centre RAPSODEE, Campus Jarlard, F-81013 Albi cedex 09, France \\ ${ }^{\mathrm{f}}$ Dept of Chemistry, S. B. College, Changanacherry, Kottayam, Kerala, India \\ ${ }^{g}$ International and Inter University, Centre for Nanoscience and Nanotechnology, Mahatma Gandhi University, Priyadarshini Hills, Kottayam, Kerala, India
}

Keywords:

Organically modified montmorillonite

Natural rubber

Nitrile rubber

Stretched-exponential relaxation

Stress relaxation

Immiscible rubber blends

\begin{abstract}
A B S T R A C T
To reduce material consumption, it is important to have reinforced material with longer life time. Incorporation of nanoparticles to reinforce and compatibilize polymer blends is one of the widely undergoing research areas in polymer science technology. A series of natural rubber and nitrile rubber (NR/NBR) nanocomposite vulcanazite, reinforced with two different organically modified clay (OMt) were prepared. To predict the performance of a material over long periods of time, stress relaxation studies with both the reinforced systems were done. The effects of loading, blend composition, filler polarity and temperature on stress relaxation of OMt reinforced NR/NBR nanocomposites were carefully measured. Based on the stress relaxation measurements, it was observed that due to its polarity difference, 01Mt (Mt modified with dimethyl, benzyl, HT modification provided by Southern Clay Products) was preferentially located at the NBR phase while O2Mt (Mt modified with mercapto silane provided by English India Clay) had more affinity with natural rubber in the NR/NBR nanocomposites. The preferential localization of OMt has been analyzed by HRTEM. The nature of interaction of the nanoclay was found to influence the stress relaxation rate. NR/NBR nanocomposites with higher filler loading showed higher rates of relaxation rate due to the presence of more filler-filler interactions. At $70{ }^{\circ} \mathrm{C}$, the viscosity ratio was found to influence the reinforcement, and consequently relaxation rate of the 50/50 NR/NBR nanocomposites. It was found that the rearrangements of the polymer chains are dependent on the blend composition, temperature, filler/polymer interactions etc. To explain and predict observed phenomena, the stretched-exponential Kohlrausch equation and Maxwell-Weichert model were used. For both models, the experimental curve fitted well with the theoretical models.
\end{abstract}

\section{Introduction}

Blending existing polymers is a very advantageous means of developing new materials, tailored to specific market segments. The philosophy of mixing materials involves combining good properties of the

Abbreviations: OC, organically modified clay; CPN, clay polymer nanocomposite; OMt, organically modified montmorillonite; O1Mt, organically modified montmorillonitecloisite 10A; O2Mt, mercapto silane modified montmorillonite; EPDM, ethylene propylene diene monomer; NR, natural rubber; NBR, nitrile butadiene rubber; ASTM, American Society for Testing and Materials; HR-TEM-, High Resolution Transmission Electron Microscope (HRTEM).

* Corresponding author at: School of Chemical Sciences, Mahatma Gandhi University, Priyadarsini Hills P.O., Kottayam-686560, Kerala, India. Tel.: +91 4812730003, +91 4812731036; fax: +914812731002.

E-mail addresses: sabuchathukulam@yahoo.co.uk, sabupolymer@yahoo.com (S. Thomas). individual materials, while mitigating their respective detrimental characteristics. Predominantly polymers are incompatible in mixtures. Thus, a compatibilizer is often added to improve the compatibility of different polymers. Nanoparticle as nanocompatibilizers is added to improve the interfacial strength of blends, by exploiting the concept of polymer-polymer nanocomposites. Organoclay (OC) mineral has been used as a compatibilizer for incompatible polymers, and it was pointed out by Rafailovich et al. (2002)that it could be added into polymer blends in order to improve the compatibility between two incompatible polymers, and to develop a more homogeneous system. The use of montmorillonite (Mt) as a reinforcing and as compatibilizing material for nitrile butadiene rubber (NBR)/styrene butadiene rubber (SBR) nanocomposites has been studied. At higher clay mineral concentration, the OC mineral was not homogeneously distributed in the matrix and agglomeration of the clay mineral causes phase separation (Essawy and El-Nashar, 2004). The influence of 
clay mineral modification on the structure and mechanical properties of Mt/EPDM nanocomposite showed that the OMt layers were fully exfoliated in the EPDM matrix and have good mechanical properties (Zheng et al., 2004).

The determination, of mechanical properties like tensile strength and elongation at break, provides us with a fundamental understanding about the behavior of a material and these experiments are timeindependent. However, these experiments are not enough to predict its long term behavior. Therefore to design a material which has good durability, it is very important to analyze the time-dependent viscoelastic nature of polymers such as stress relaxation, creep, dynamic mechanical properties etc. The stress relaxation experiment is conducted in order to characterize the time-dependent reinforcement of clay polymer nanocomposite (CPN) (Le et al., 2010). The viscoelastic rubber, when strained at a constant rate to a fixed deformation, the stress required to maintain that strain decays with time. This is referred to as stress relaxation. The stress relaxation rate depends upon the probability of transition of the system from one stage of equilibrium to another (Tager, 1972). It may be a one-stage or two-stage mechanism in multiphase systems (AbuAbdeen, 2010). The processes that can occur during stress relaxation of a typical rubber vulcanizate include physical relaxation, which occurs due to the rearrangement of the polymer chains and the fillers when subjected to deformation and the chemical effect which is more prominent at higher temperature and at longer durations due to the aging of rubber by oxidative chain scission, crosslink breakage, or crosslink formation (Abu-Abdeen, 2010).

To this date, only few studies reported about the time and temperature dependent reinforcement of CPN, especially in rubber blend systems (Shah et al., 2005; Fritzsche et al., 2008; Fritzsche et al., 2009). The stress relaxation behavior of short-jute-fiber-rein-forced acrylonitrile butadiene rubber composites studied by Bhagawan et al. (1987) reported that a two-stage relaxation pattern existed in these composites. Flink and Stenberg (1990) reported that stress relaxation studies could provide insight into the interfacial adhesion in NR composites reinforced with short cellulose fibers. Varghese et al. (1994) studied the stress relaxation behavior of acetylated short-sisal-fiber-reinforced NR composites. Recently, from this laboratory, Meera et al. (2009) investigated the tensile stress relaxation behaviour of natural rubber reinforced with titania and nanosilica. The rate of stress relaxation was found to increase with increase in filler loading for both the filled system and the silicafilled system, showing a higher rate of stress relaxation compared to the $\mathrm{TiO}_{2}$-filled NR. Thus it is understood that the relaxation mechanism in nanocomposites is dependent on many factors, such as the nature of the polymers, filler and interface, strain level, strain rate, and temperature.

The rate of stress relaxation of rubber chains can be influenced by its surrounding structure and will be different from other chains even in a homogeneous, unfilled rubber compound. When one compares a polymer/polymer blend nanocomposite and a CPN, a major difference is observed between both morphological and thermomechanical properties. As with the behavior during the deformation stage, the behavior will be different during the relaxation stage also. The complexity is increased by the presence of nanoparticles. Different types of relaxation process can take place under loading, which will vary with the different types of interfaces in the material. Naturally, this variation in deformation was expected to result in different modes of stress relaxation behavior. Two OMt were used for the blend preparation. One was cloisite 10A which is polar (O1Mt) and the other was Nanocaliber $200 \mathrm{~m}$ which is nonpolar (O2Mt).

Present work, is to find out the effect of filler loading, blend composition and nature of OMt material on the stress relaxation behavior of NR/NBR nanocomposites. We have reported here the stress relaxation behavior of the OMt filled elastomer blends of natural rubber and nitrile rubber. Both NR and NBR have specific individual properties like good mechanical properties for NR and good oil resistance for NBR. Incorporation of OMt into this elastomeric blend was done as an attempt to make the two rubbers compatible. OMt was found to be effective in compatibilizing elastomer blends (Rafailovich et al., 2002). It appears, no work has been reported explaining the effect of OMt on the stress relaxation behavior of NR/NBR blend nanocomposites.

\section{Materials and methods}

Polymers used in this study were natural rubber (NR) and nitrile rubber (NBR). Natural rubber ISNR 5 was supplied by the Rubber Board, Kottayam, Kerala, India The weight average molar mass of NR was $\mathrm{Mw}=780,000$ and Mooney Viscosity $85 \mathrm{ML}(1+4)$ at $100{ }^{\circ} \mathrm{C}$. Nitrile butadiene rubber (NBR), with $33 \%$ acrylonitrile content and having Mooney viscosity of $\mathrm{ML}(1+4)$ at $100{ }^{\circ} \mathrm{C}$ and a specific gravity of about 0.98 approximately, was supplied by the Eliokem Industries Ltd. Mumbai. The two OMt used for the study, Cloisite 10A and Nanoclaiber $200 \mathrm{~m}$, were provided by the Southern Clay Products, Mumbai India and the English India Clay, Thiruvananthapuram Kerala, India respectively. Cloisite $10 \mathrm{~A}$ is an organically modified Mt with dimethyl, benzyl, one alkyl tail ie hydrogenated tallow HT (65 m\% C18, 30 m\% C16, 5 m\% C14 m\%) modification with cation exchange capacity (CEC) equal to $125 \mathrm{meq} / 100 \mathrm{~g}$ and an average dry particle size was in the range $2 \mu \mathrm{m}-13 \mu \mathrm{m}$. Hereafter, cloisite $10 \mathrm{~A}$, will be denoted in this paper as 01Mt. Nanoclaiber $200 \mathrm{~m}$ is an organically modified Mt with Mercapto silane modification with $60-80 \mathrm{~nm}$ average in particle thickness, $200 \mathrm{~nm}$ average in diameter and specific surface area of $3.06 \mathrm{~m}^{2} / \mathrm{g}$. The content of this OMt was $45 \%$ silica, alumina $38 \%$, Fe- $0.5 \%$,TiO2$5.5 \%, \mathrm{CaO}-0.6 \%, \mathrm{MgO}-0.07 \%, \mathrm{Na}_{2}-0.1 \%, \mathrm{~K}_{2} \mathrm{O}-0.03 \%$. The silane content for modification was $0.5 \%$. This nonpolar OMt will be denoted as $02 \mathrm{Mt}$ hereafter in this document. The compounding ingredients (Table 1) used were of commercial grade.

\subsection{Compounding and curing}

\subsubsection{Preparation of NR/NBR nanocomposite}

The NR/NBR nanocomposites were compounded according to the formulation given in Table 1 with the aid of a laboratory-sized two roll mixing mill $(150,300 \mathrm{~mm})$ according to ASTM D-3182. The nip gap, roll speed ratio, and number of passes were kept the same for all of the mixes. The temperature range for mixing was $60-90{ }^{\circ} \mathrm{C}$. Both the rubbers were masticated for 2-3 min. They were mixed together to get a homogenous sheet. The compounding ingredients and OMt were then added (Table 1). The different formulations prepared are given in Table 2. After mixing, the rubber composition was molded in an electrically heated hydraulic press to optimum cure, using moulding conditions that were previously determined from rheometer torque data. All the compositions represented in the paper follow the order NR/NBR (Table 2). Stress relaxation specimens were taken in accordance with ASTM D $638 \mathrm{M}$ standard. (1: $200 \mathrm{~mm}$, t: $4 \mathrm{~mm}$, type 1 ).

\section{Table 1}

Compounding ingredients used ${ }^{\mathrm{a}}$

\begin{tabular}{ll}
\hline Materials & Parts per hundred $(\mathrm{phr})$ of rubber (in the order of mixing) \\
\hline Zinc oxide & 2.5 \\
Stearic acid & 1.5 \\
OMt $^{\mathrm{b}}$ & varied from $0 / 1 / 2 / 5 / 10$ \\
TMTD $^{\mathrm{c}}$ & 0.2 \\
CBS $^{\mathrm{d}}$ & 1.3 \\
Sulphur & 2.25 \\
\hline
\end{tabular}

a Amount of ingredients expressed in per hundred rubber,(phr).

b Organically modified montmorillonite-OIMt \&02Mt.

c Tetra methyl thiurum disulphide.

d $\mathrm{N}$-cyclohexyl benzothiazyl sulphanamide. 
Table 2

Formulations of the blend.

\begin{tabular}{lllll}
\hline Materials & $100 / 0$ & $70 / 30$ & $50 / 50$ & $0 / 100$ \\
\hline NR & 100 & 70 & 50 & 0 \\
NBR & 0 & 30 & 50 & 100 \\
${ }^{*} 01 \mathrm{Mt}^{\mathrm{a}}, 02 \mathrm{Mt}^{\mathrm{b}}$ & $0 / 1 / 2 / 5 / 10$ & $0 / 1 / 2 / 5 / 10$ & $0 / 1 / 2 / 5 / 10$ & $0 / 1 / 2 / 5 / 10$ \\
\hline
\end{tabular}

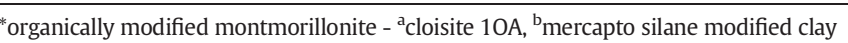

\subsection{Characterisation}

\subsubsection{Stress relaxation measurements}

Stress relaxation measurements were carried out to study the effect of different parameters that influence the NR/NBR nanocomposite such as loading, filler polarity, blend composition, mixing conditions, nature of filler, temperature and preparation methods. A Tinius Olsen testing machine (H50KT) fitted with an environmental chamber was used to carry out the viscoelastic testing. The environmental chamber was capable of temperature control. After the environmental chamber was equilibrated to the desired temperature $\left(70{ }^{\circ} \mathrm{C}\right)$, the specimen was gripped between two sets of jaws $12 \mathrm{~mm}$ apart and then was further allowed to equilibrate to the conditions within the chamber, for a period of $30-40 \mathrm{~min}$. Once equilibrated, a tensile stress was applied to the specimen by moving the upper grip at a constant rate $\left(500 \mathrm{~mm} \mathrm{~min}^{-1}\right.$ crosshead speed). When the desired initial strain of $50 \%$ had been reached, movement of the grip was halted and the decrease in stress with time (relaxation) was recorded for a period of $90 \mathrm{~min}$.

The dumbbell specimens were also extended to different strain levels at different strain rates. When appropriate strain of 50\% was reached, it was maintained, and the stress was recorded for a time span of $90 \mathrm{~min}$. Because stress relaxation is exponential, the decrease in stress is very prominent during the initial period of relaxation, and therefore, stress monitoring was started immediately after the required strain was attained.

\subsubsection{Transmission electron microscopy}

To assess the quality of filler dispersion and morphological details, the NR/NBR nanocomposites were investigated by means of transmission electron microscopy (TEM) (JEM-2100 HRTEM). The micrographs were obtained in point to point resolution $0.194 \mathrm{~nm}$, operating at an accelerating voltage of $200 \mathrm{kV}$. Cryocut specimens prepared using an ultra-microtome (Leica, Ultracut UCT) were placed on a Cu grids of 300 mesh (35 mm diameter) and were analysed.

\subsection{3. $X$-ray diffraction $(X R D)$ analysis}

XRD of NR/NBR nanocomposite was conducted using the XRD: SIEMENS D 5000 with radiations $\mathrm{Cu} \mathrm{K}$ alpha at $40 \mathrm{kV}$ and $30 \mathrm{~mA}$.

\section{Results and discussion}

\subsection{Effect of nature of filler}

Two types of blend compositions (70/30 and 50/50 NR/NBR) were taken for the study to differentiate the interaction of fillers, O1Mt and O2Mt with the two elastomers. The stress relaxation plots of 70/30 and 50/50 NR/NBR nanocomposites with O1Mt and O2Mt are given in Fig. 1. For better understanding of the slope of each system, a plot of slope vs different filler is shown in the inset. It is understandable from Fig. 1 that the initial and final stress value for both 70/30 and 50/50 NR/NBR nanocomposite with O2Mt modified clay mineral is higher compared to the other NR/NBR nanocomposites (Fig. 1). The slope of the relaxation curve of 50/50 and 70/30 NR/NBR (Fig. 1) is found to be steeper for 01Mt loaded NR/NBR nanocomposites than that for O2Mt filled NR/NBR nanocomposites.(Fig. 1 inset). The improved mechanical property and the slow stress relaxation of O2Mt filled NR/NBR filled nanocomposites are due to the strong interaction between 02Mt and NR. Due to its non polar nature, O2Mt goes to the NR phase preferentially. Modifications such as mercapto and organofunctional silane can act as molecular bridges between NR molecules and clay mineral particles. Thus O2Mt creates strong crosslinks with the NR phase during vulcanization. The intercalated $02 \mathrm{Mt}$ results in good dispersion and enhances the polymer/filler interactions. Additionally, the electron microscopy data also support the fact that, O2Mt preferentially goes to the NR phase (Fig. 2). Fig. 3 represents the hypothesis of interaction between $02 \mathrm{Mt}$ and the two rubbers.

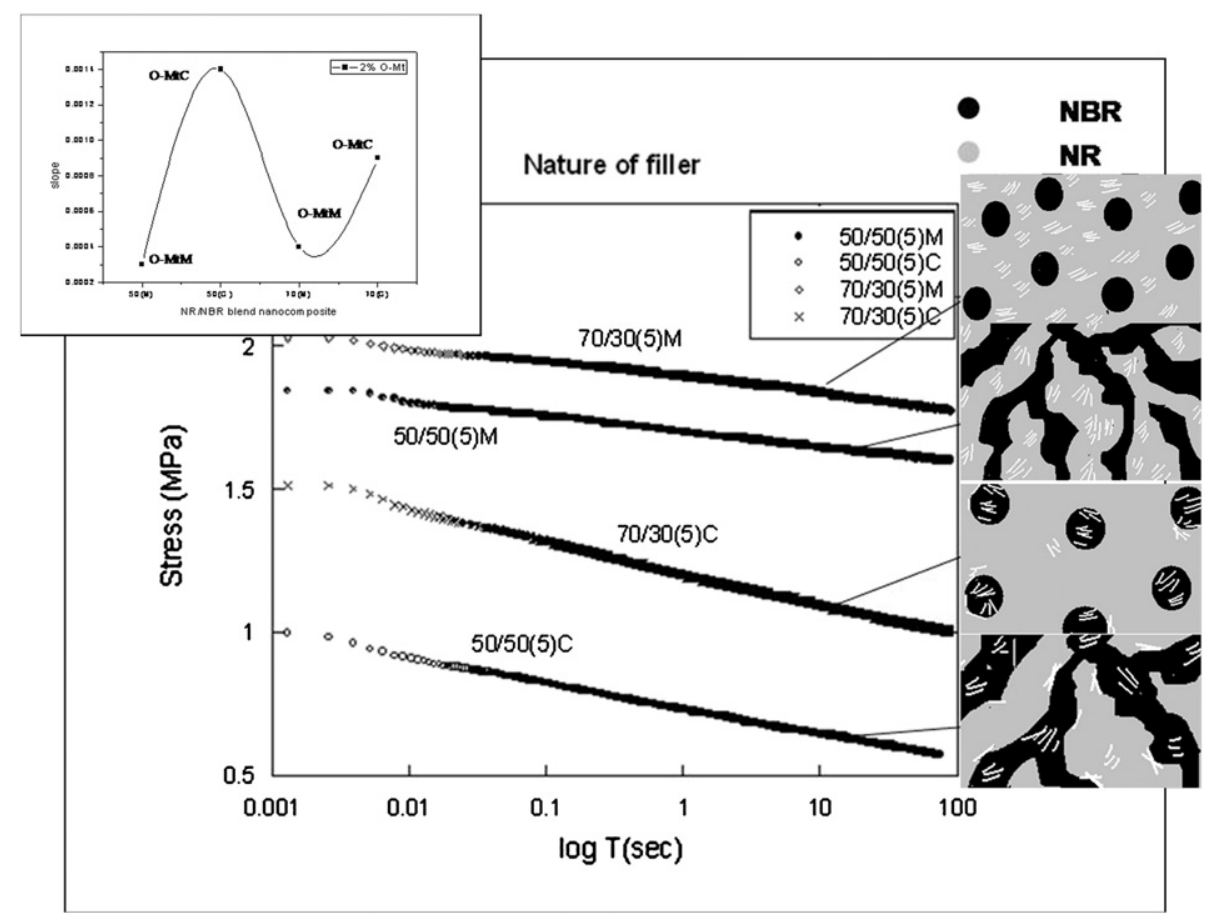

Fig. 1. Effect of nature of filler on the relaxation behavior of NR/NBR blend nanocomposite. Schematic representation shows the preferential migration of the two O-Mts towards the polymers. The figure in the inset shows the plot of slope vs filler loading. (C-Cloisite10A, M-mercapto silane modified clay). 


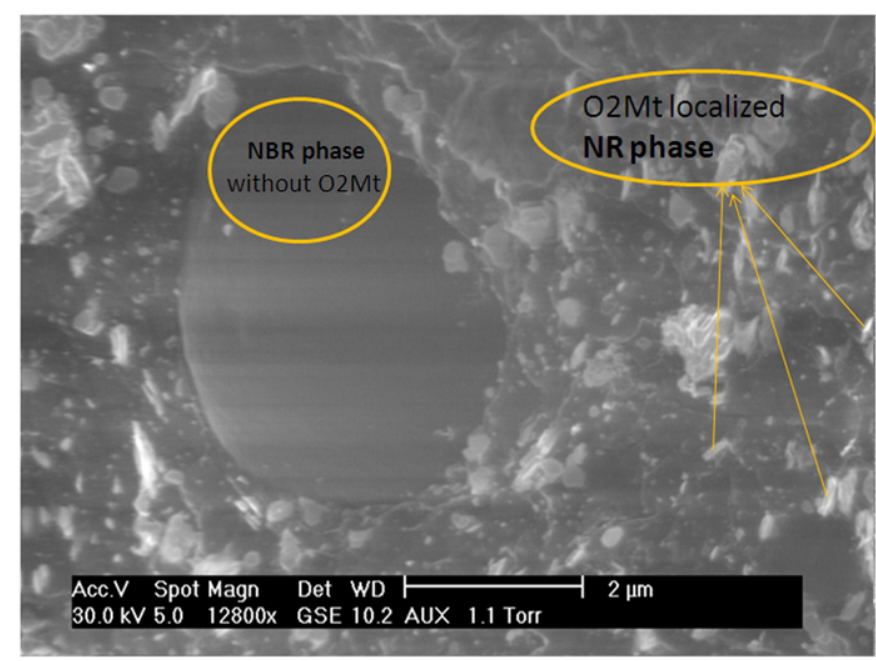

Fig. 2. SEM images showing the O2Mt clay localized in the NR phase.

The viscosity of the NR phase will increase as more number of O2Mt goes to this phase. It is generally accepted that viscosity ratio [ie the ratio of viscosity of the dispersed phase to the viscosity of the matrix $(\lambda)]$ is one of the most critical variables for controlling blend morphology. Researchers (Aerts and Everaert, 1999; Favis and Chalifoux, 1987; Loan and Emmanuel, 2007) reported that low viscosity ratio is more favorable for a finer morphology, because high viscous matrix enhances droplet break up by exerting an efficient shear stress transfer towards the dispersed phase, leading to finer diameter of the dispersed phase. Also, it has been already reported that once the viscosity of the matrix phase is increased, the domain size of the dispersed phase will be decreased due to rheological reasons (Paul and Bucknall, 2000). For a viscoelastic dispersion, the critical condition for droplet breakup is given by (Choi et al., 1996)

$\mathrm{W}_{\mathrm{e}} \geq \frac{\mathrm{F}\left(\frac{\eta \text { dispersed }}{\eta \text { matrix }}\right)}{\sin (2 \varphi)}$

where $W_{\mathrm{e}}$ is Weber number related to the viscosity ratio, $\eta_{\text {dispersed }}$ is the viscosity of the dispersed phase, $\eta_{\text {matrix }}$, viscosity of matrix phase, $\mathrm{F}$ is an empirical function of the viscosity ratio and $\Phi$ the orientation angle. The lower the viscosity ratio the larger the deformation of NBR domains ie when the viscosity ratio is smaller than unity, the domain size of the dispersed NBR phase is finer and its distribution is more uniform. Thus for $70 / 30 \mathrm{NR} / \mathrm{NBR}$, the preferential localization of O2Mt in the NR phase will increase the NR phase viscosity, leading to a fine and uniform distribution of NBR domain in the NR continuous phase. Since the molar mass of the NR phase is extremely high, the complete migration of the clay mineral platelets of the clay mineral into NR phase will be restricted

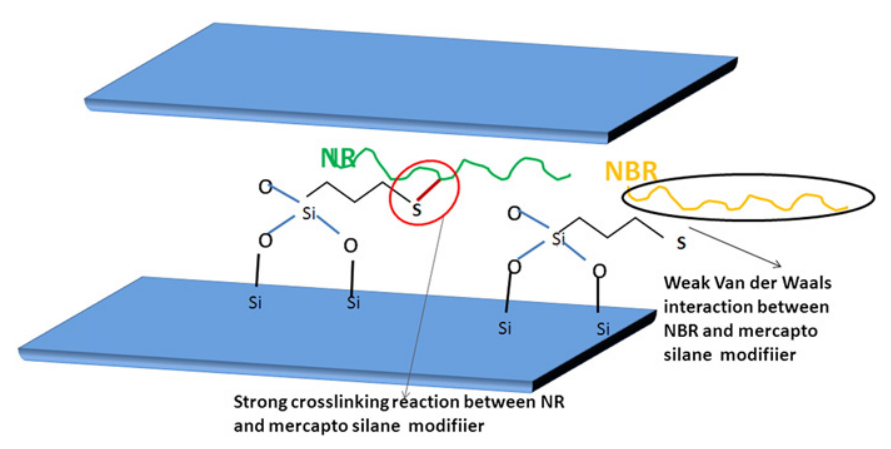

Fig. 3. The hypothesis of interaction between O2Mt and NR. by diffusional constraints. Therefore, it is reasonable to believe that a substantial portion of the clay mineral will stay at the interface. Fig. 3 explains the type of interactions in O2Mt NR/NBR nanocomposite. The slow relaxation behavior of NR/NBR nanocomposites for the 70/30 NR/NBR containing O2Mt system is thus due to the strongly bonded O2Mt to the NR phase. For the 50/50 NR/NBR with O2Mt system too, the clay mineral is localized at the NR and at the interface. Interestingly, this system also shows very slow relaxation process as explained earlier.

On the other hand, 01Mt shows affinity towards NBR predominantly. This can also be established from the TEM images(Fig. 4a, b \& c). The polar interaction between NBR and O1Mt, localizes the O1Mt, predominantly in the NBR phase. This results in the increase of NBR phase viscosity, which ultimately decreases the polymer chain mobility and suppresses the coalescence of the NBR domains, leading to a fine morphology in the NR matrix for the 70/30 NR/NBR system.(Fig. 5). According to viscosity ratio, the increase in viscosity of the dispersed NBR phase will not favour a fine morphology. However, in this situation the decrease in dispersed domain size is also attributed to the comparable viscosity of NR and NBR phase as a result of the localization of O1Mt in the NBR phase (the pure NR viscosity is much higher than NBR viscosity). The presence of 01Mt at the interface of the two elastomers, (Fig. 4a) shows the partial interaction of 01Mt towards NR phase. The alkyl rich HT present in 01Mt imparts the interaction between NR and 01Mt. The presence of HT in 01Mt slightly weakens the polar interaction between 01Mt and NBR (Hotta and Paul., 2004; Shah et al., 2005; Shah and Paul, 2006). Consequently, the OMt acts as an amphiphile holding the two rubbers. The strength of interaction to either of the elastomer is estranged. As a result of these weak bonds, the relaxation takes place extremely fast in the case of 01Mt filled systems. The mechanical property shown in Table 3 confirms the presence of comparatively stronger interaction in the case of O2Mt filled NR/NBR nanocomposites.

It is also important to point out that in all cases, a two stage relaxation process is observed. The relaxation at the initial phase relates to the filler-filler rupture and the second phase corresponds to breakage of the polymer/filler and polymer/polymer interactions. That is, interaction with different interfaces in the NR/NBR nanocomposites relaxes at different rates.

\subsection{Effect of filler loading}

As the filler loading is increased the relaxation rate also was found to increase. The rate of stress relaxation will be more when there is more number of interactions that lead to rupture, resulting in an increase in entropy. As reported earlier (Geethamma et al., 2004), the conditions favoring an increase in entropy will enhance the rate of stress relaxation. So an increase in slope corresponds to rupturing or relaxation of these interactions. The initial stress value was found to be increasing with increase in 01Mt loading (Fig. 6). This can be due to the more number of filler/filler interactions in the case of higher filler loaded samples corresponding to an improved reinforcing effect. However, as the filler content is increased, an increase in slope of the stress relaxation curve was observed. Although an improved reinforcement is shown initially due to a higher number of interactions, most of these filler-filler interactions were easily ruptured. The lower residual stress value observed in the case of $10 \mathrm{phr}$ 01Mt loaded NR/NBR nanocomposites may also be associated with the breakage of these weak filler/filler and polymer/filler networks during the course of relaxation process (Ray et al., 2002). It was interesting to note that $2 \mathrm{phr}$ NR/NBR nanocomposite has better reinforcement than $5 \mathrm{phr}$ loaded sample, as the former showed higher initial and residual stress value. This was supported by the XRD and TEM results (Figs. 7 and $4 \mathrm{c} \& \mathrm{~d}$ ) where we can observe a fairly better intercalated and exfoliated morphology for $2 \mathrm{phr}$ NR/NBR nanocomposites with 01Mt as the filler, while higher filler loaded samples indicated the presence of intercalated aggregate structure. The fact that $1 \mathrm{phr}$ polymer polymer nanocomposite shows an intercalated aggregate structure may be because of the isolated clay mineral 

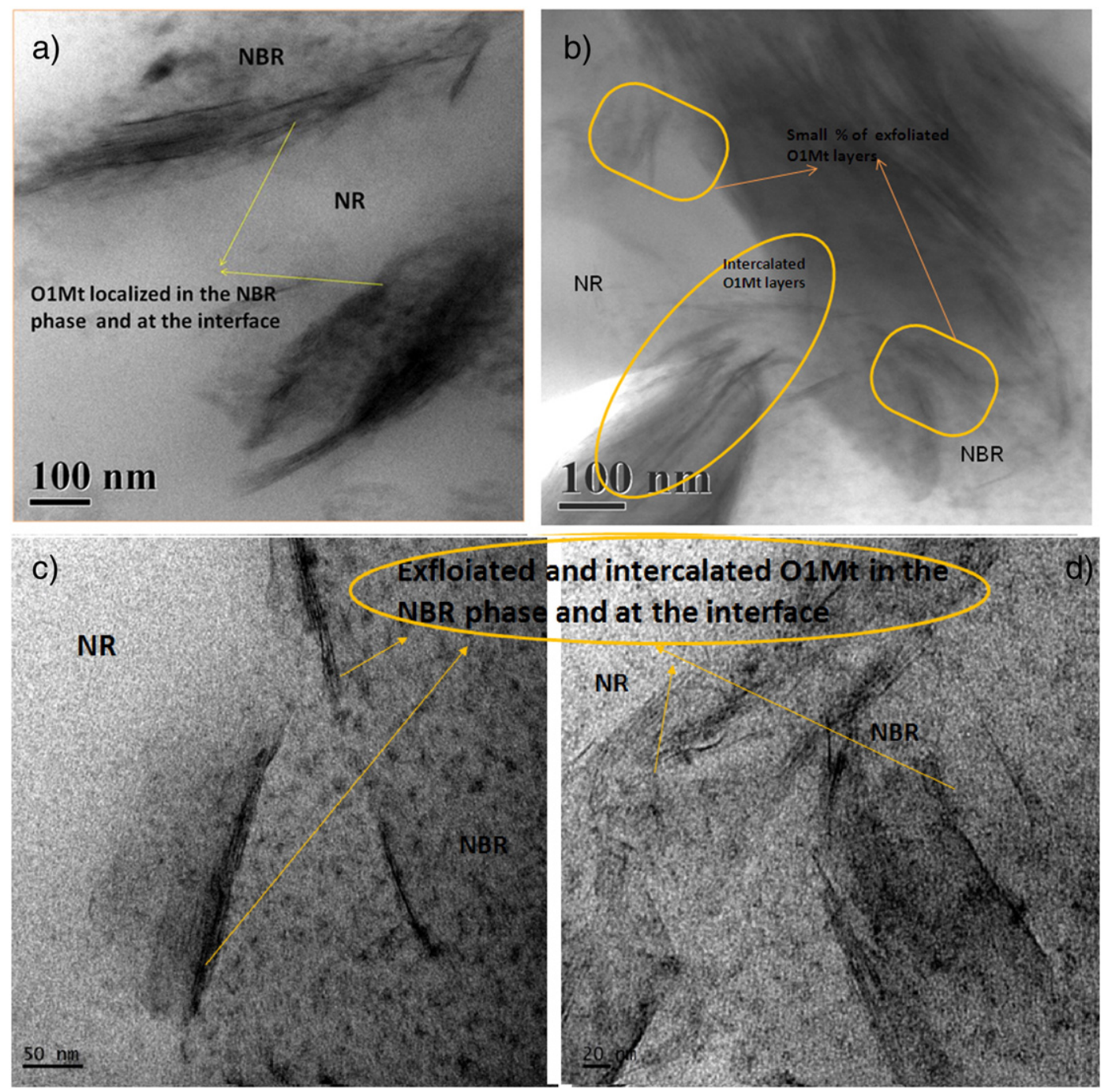

Fig. 4. TEM image showing the localization of O1Mt in 50/50 blend nanocomposites a) \& b) in the NBR phase and at the interface c) \& d) the intercalated and exfoliated morphology of OImt.

aggregates in the rubber matrix with low filler loading. Also at $1 \mathrm{phr}$ 01Mt loading shows low initial reinforcement compared to higher filler loading because in this bad dispersion state, the contact area between rubber and clay mineral is insignificant, and therefore, clay mineral shows only a minimal reinforcement effect (Freeman et al., 2001). Agglomeration at higher concentration should have resulted in a higher relaxation rate due to more and more filler/filler rupturing. Intercalation of polymers between the 01Mt layers and the subsequent rupturing of this interaction on stress relaxation is represented in Fig. 6. The two stage mechanism observed in this case also suggests the different types of interfaces existing in the polymer/polymer nanocomposites. The initial portion represents
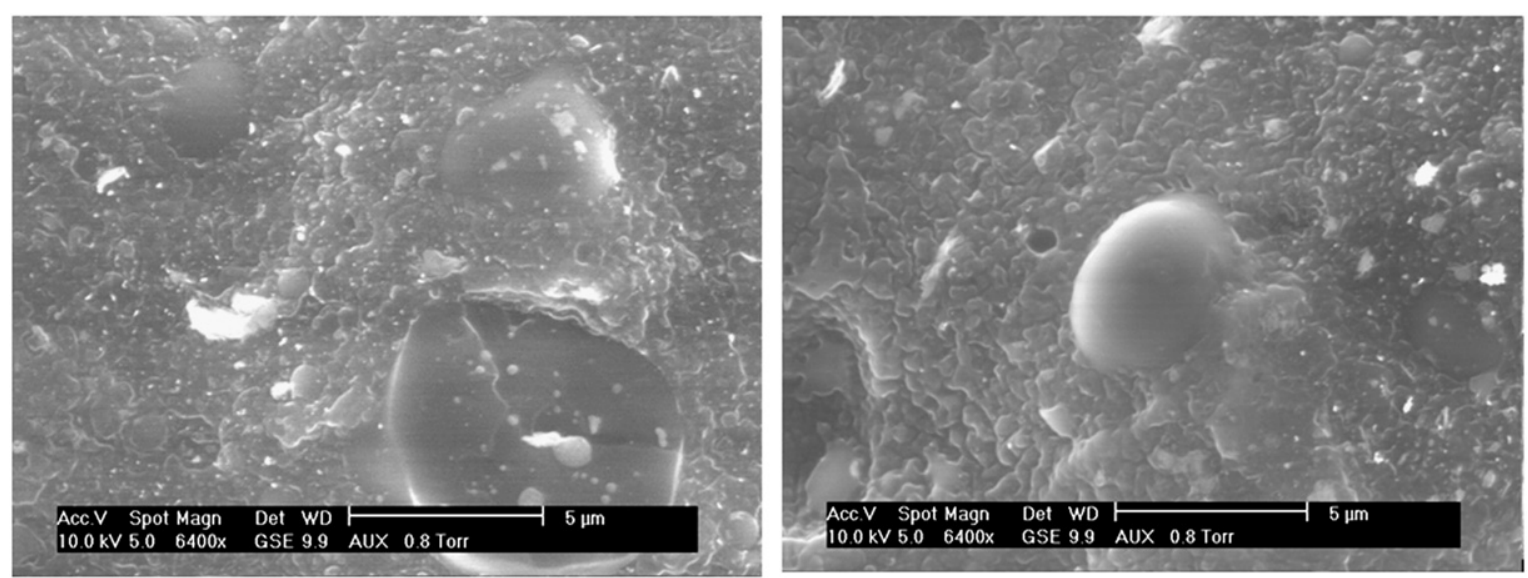

Fig. 5. SEM images showing the decrease in domain size of NBR dispersed phase. 
Table 3

Tensile properties of NR/NBR blend nanocomposites with the two different fillers.

\begin{tabular}{lcccccc} 
& $70 / 30(0)$ & $70 / 30(5) \mathrm{M}$ & $70 / 30(5) \mathrm{C}$ & $50 / 50(0)$ & $50 / 50(5) \mathrm{M}$ & $50 / 50(5) \mathrm{C}$ \\
\hline${ }^{\mathrm{a}}{ }_{\sigma_{\mathrm{b}}}(\mathrm{MPa})$ & 12.91 & 21.3 & 10.93 & 3.28 & 13.42 & 5.75 \\
${ }^{\mathrm{b}} \varepsilon_{\mathrm{b}(\%)}$ & 226 & 530 & 4.96 & 333 & 416 & 331 \\
${ }^{\mathrm{c}} \sigma_{100}(\mathrm{MPa})$ & 1.68 & 1.23 & 0.78 & 0.67 & 1.23 & 0.88 \\
${ }^{\mathrm{d}}{ }_{\sigma_{200}}(\mathrm{Mla})$ & 9.783 & 4.46 & 1.57 & 1.30 & 2.32 & 1.81 \\
\hline
\end{tabular}

M-Mercapto silane modified organoclay

C-Cloisite 10A

a Tensile strength.

b Elongation at break (\%).

c Stress at $100 \%$.

d Stress at $200 \%$.

breakage of the filler/filler interactions, which can be observed clearly from the curve of $10 \mathrm{phr}$ NR/NBR nanocomposite, where the slope of the initial portion is higher. It should also be pointed out that the relaxation for NR/NBR blend without $01 \mathrm{Mt}$ is lower than that for NR/NBR nanocomposites. This may be explained based on the effect of orientation of OMt and polymer chains. However, this has to be reexamined further using techniques like small angle X-ray diffraction studies and will be produced in the next paper.

\subsection{Effect of temperature}

The stress relaxation behavior at higher temperature was studied by comparing the stress relaxation plot of 50/50 and 70/30 NR/NBR loaded with $2 \mathrm{phr} 01 \mathrm{Mt}$ at room temperature $\left(30^{\circ} \mathrm{C}\right)$ and an elevated

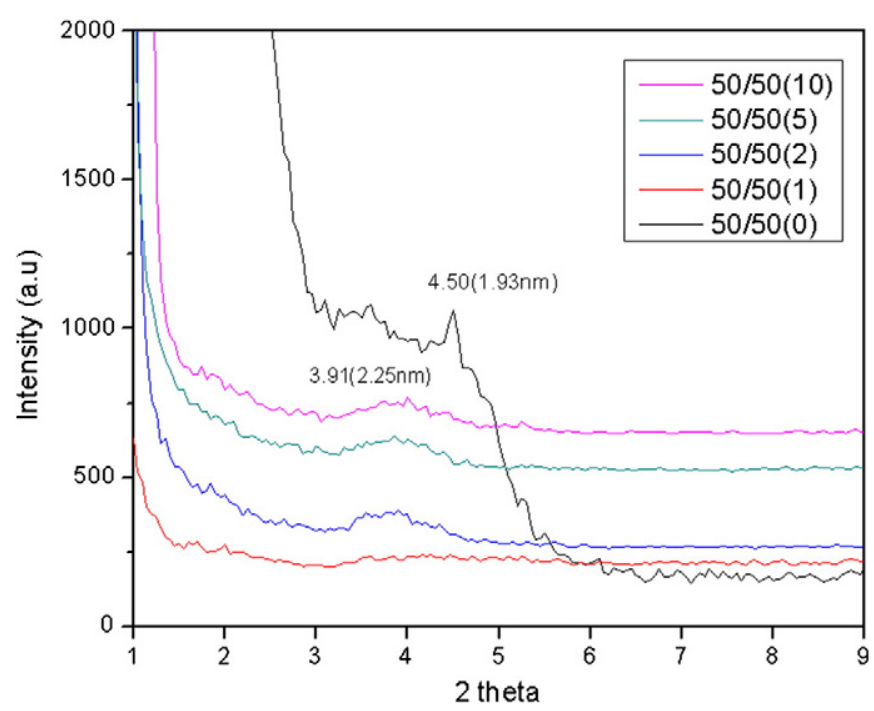

Fig. 7. XRD pattern of 50/50 blend with different O1Mt loading.

temperature $\left(70^{\circ} \mathrm{C}\right)$ (Fig. 8). In addition to that, a plot variation in slope value with temperature for different nanocomposites is shown in the inset. At $70{ }^{\circ} \mathrm{C}$, even though a higher initial stress value (Fig. 9) was shown by 50/50 (2) NR/NBR nanocomposite, the slope of the curve for 70/30(2) was found to be lower (Fig. 8). Variation in slope value with temperature for different polymer/polymer nanocomposites

\section{Effect of filler loading}

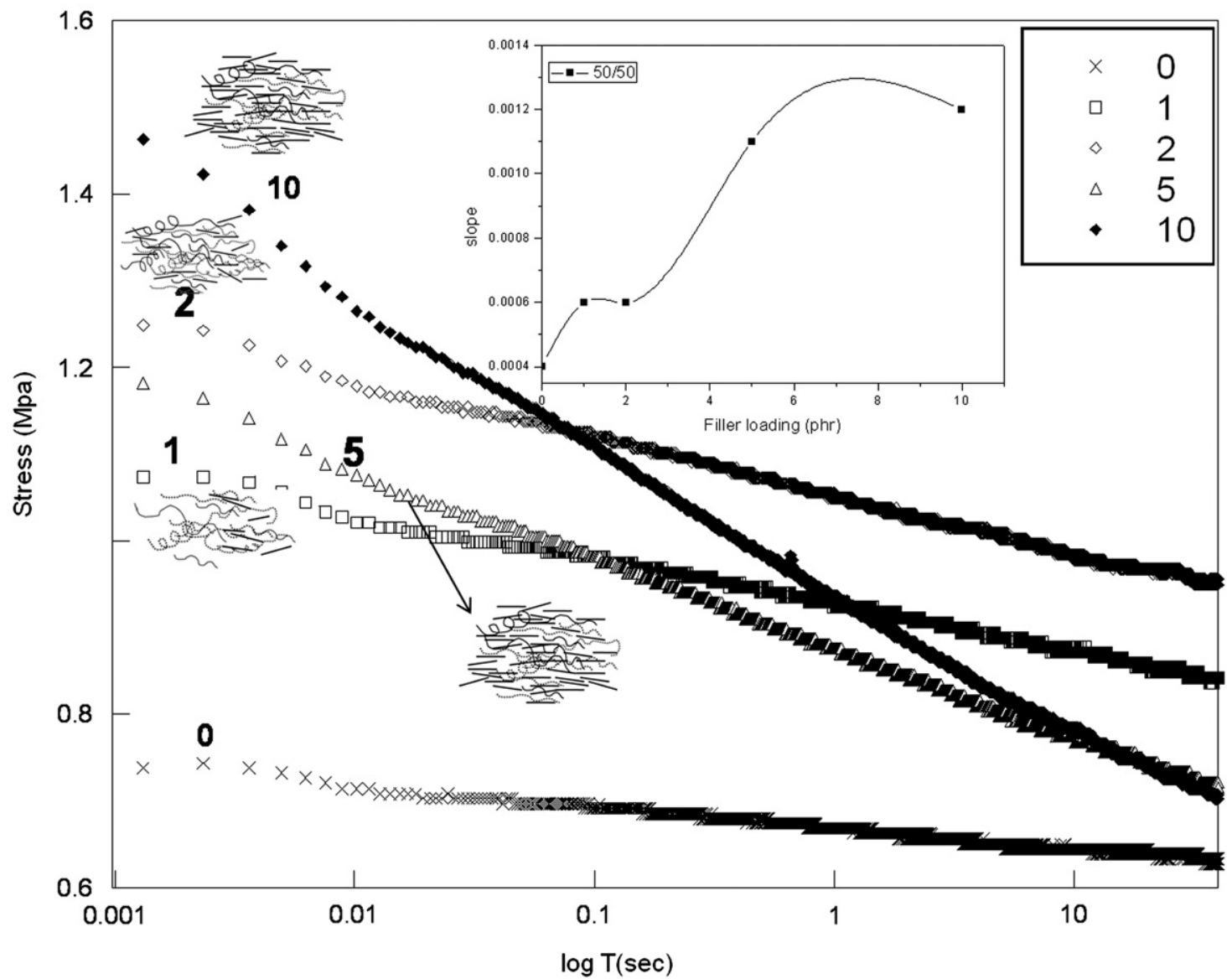

Fig. 6. Effect of filler loading on stress relaxation behavior of 50/50 NR/NBR blend nanocomposite with O1Mt.The schematic representation shows the intercalated aggregates of the O1Mt at different filler loading. In the inset the plot of slope vs filer loading is given. 


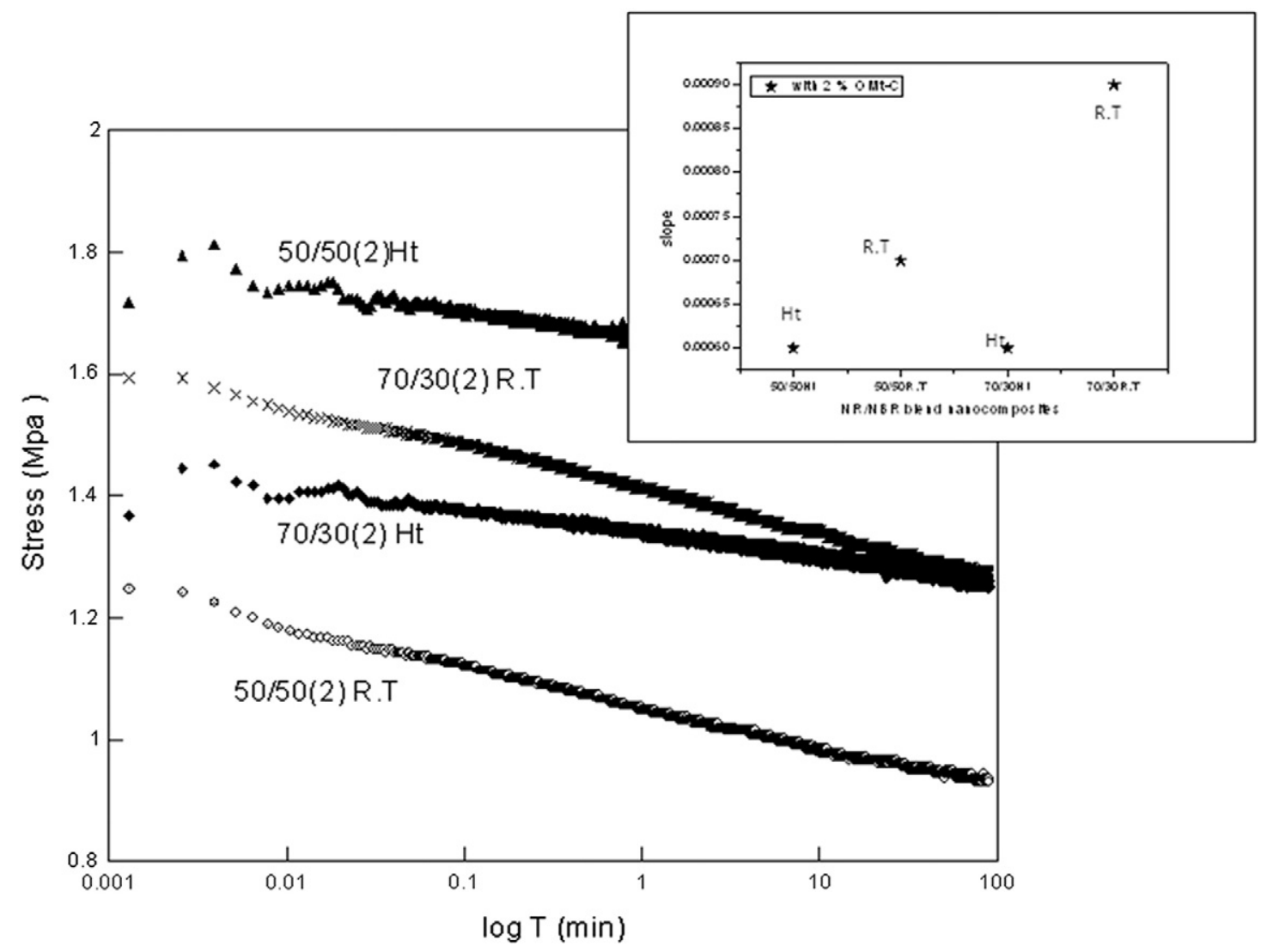

Fig. 8. Stress relaxation curves of two different blend compositions of NR/NBR blend at two different temperatures. Inset, the slope of the nanocomposites at different temperature.

is shown in the inset. This can be explained on the basis of the irreversible changes occurring in the system and of viscosity (Cao and Sadhan, 2007). Irreversible changes occurring in the system like increased bonding between clay mineral and polymer, increased crosslinks etc can make the interactions more stable. It has been reported earlier, that when the viscosity of the matrix is very high $\eta_{\mathrm{d}} / \eta_{\mathrm{m}}<<1$, then the viscosity factors outweigh the interfacial tension (Loan and Emmanuel, 2007) and the presence of OMt at the interface will not affect the viscosity ratio. In this context, if we analyze the 70/30 and 50/50 NR/NBR 01Mt system, in the former case, at room temperature, majority of 01Mt may be in the NBR phase due to polarity factors, and slightly at the interface due to its

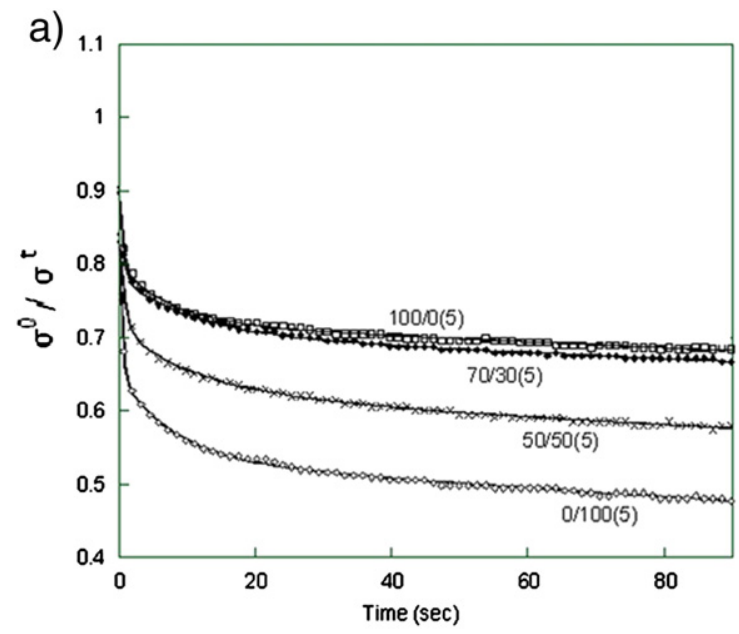

affinity towards NR as a result of the alkyl groups in hydroxy tallow. (ie $\eta \mathrm{d} \gg \gg>\mathrm{\eta m}$ ). At $70^{\circ} \mathrm{C}$, a major change in viscosity can be shown only by the matrix phase, as variation in the viscosity of the highly filled dispersed phase is difficult at this temperature. This decrease in viscosity of NR matrix phase can pull the 01Mt from the interface towards it, resulting in a better interaction between NR and $01 \mathrm{Mt}$ at $70^{\circ} \mathrm{C}$. The fact that polar interaction between polymer and clay mineral (Meeting of the American Chemical Society, Cleveland, Ohio, 1914) will weaken at higher temperature drives the migration of 01Mt towards the NR phase. This new interaction between NR and O1Mt causes a decrease in relaxation rate for both the composition at higher temperature. At higher

b)

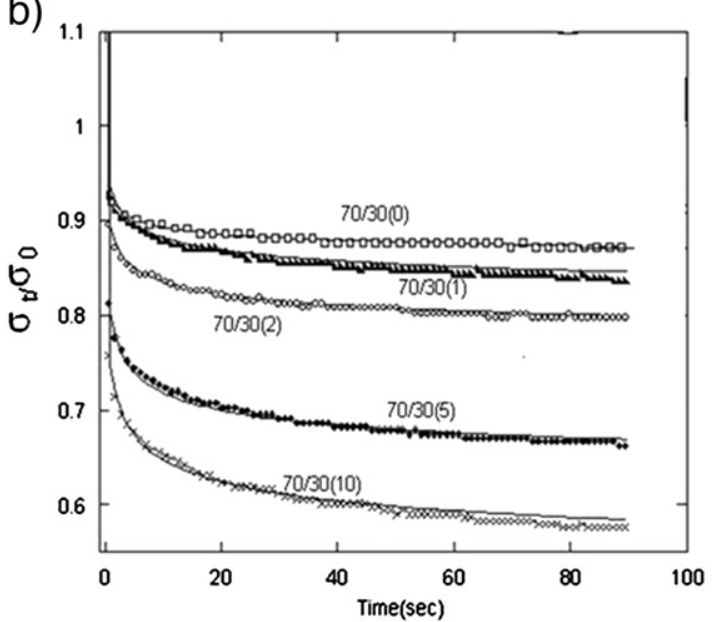

Fig. 9. Time dependence of normalized stress for a) different NR/NBR blend nanocomposites with Cloisite 10A and b) different C10A loaded 70/30 NR/NBR blend (curve fitting with Kohlrausch model). 
Table 4

Curve fitting results for stress relaxation data according to Fig. 9a and b.

\begin{tabular}{lllllcl}
\hline Composition & $\sigma_{\circ} / \sigma_{0}$ & $\sigma_{1} / \sigma_{2}$ & $\mathrm{~B}$ & $10^{3} / \mathrm{s}$ & $\mathrm{X}^{2}$ & $\mathrm{R}$ \\
\hline $\mathbf{1 0 0} / \mathbf{0}(\mathbf{5})$ & 0.659 & 0.277. & 0.135 & 0.057 & 1.365 & 0.985 \\
$\mathbf{7 0} / \mathbf{3 0}(\mathbf{5})$ & 0.641 & 0.261 & 0.144 & 0.145 & 1922 & 0.983 \\
$\mathbf{5 0} / \mathbf{5 0}(\mathbf{5})$ & 0.537 & 0.293 & 0.142 & 0.184 & 4.367 & 0.975 \\
$\mathbf{0} / \mathbf{1 0 0}(\mathbf{5})$ & 0.445 & 0.311 & 0.165 & 0.222 & 2.153 & 0.990 \\
$\mathbf{7 0} / \mathbf{3 0}(\mathbf{0})$ & 0.855 & 0.175 & 0.092 & 0.010 & 1.160 & 0.940 \\
$\mathbf{7 0} / \mathbf{3 0}(\mathbf{1})$ & 0.794 & 0.231 & 0.093 & 0.041 & 3.120 & 0.930 \\
$\mathbf{7 0} / \mathbf{3 0}(\mathbf{2})$ & 0.767 & 0.230 & 0.1101 & 0.042 & 2.070 & 0.960 \\
$\mathbf{7 0} / \mathbf{3 0}(\mathbf{5})$ & 0.641 & 0.261 & 0.1445 & 0.145 & 1.920 & 0.980 \\
$\mathbf{7 0} / \mathbf{3 0}(\mathbf{1 0})$ & 0.535 & 0.291 & 0.1453 & 0.193 & 3.440 & 0.980 \\
\hline
\end{tabular}

temperature, there is the possibility of additional crosslinks for both the phases, due to aging processes. This also could have resulted in the decreased relaxation rate at higher temperature.

\section{Modelling}

Two models were applied to compare the theoretical value with experimental results. One is a two component stretched exponential Kohlrausch equation (Ferry, 1970) and the other is three elements Maxwell-Weichert model (Aklonis et al., 1972) with three relaxation times. It was found that both models fitted well with the experimental stress relaxation curves. The stretched exponential Kohlrausch equation used to fit the experimental curves of the nanocomposites is given by

$\frac{\sigma_{\tau}}{\sigma_{0}}=\frac{\sigma_{\infty}}{\sigma_{0}}+\frac{\sigma_{1}}{\sigma_{0}} \mathrm{e}^{\left[-\left(\frac{\mathrm{t}}{\mathrm{\tau}}\right) \beta\right]}$

where $\sigma_{\infty} / \sigma_{0}, \sigma_{1} / \sigma_{0}$, and $\beta$ are the fitting parameters. The relaxation time $\tau$, the stretching parameter $\beta$, transient stress $\sigma 1 / \sigma_{0}$, and limiting stress $\sigma_{\infty} / \sigma_{0}$ are given in Table 4 . The extent of fit was made from the $\chi^{2}$ and $\mathrm{R}$ value, given in the table (Table 4 ) to assess the quality of the fits.

The experimental curves fitted to the above Eq. (2) are shown in Fig. 9a and b for varying composition, NR/NBR nanocomposites and for different 01Mt loaded 70/30 NR/NBR nanocomposites respectively. It is clear from the data (Table 4 ) that the limiting stress value $\sigma_{\infty} / \sigma_{0}$ decreases as the filler content is increased. One reason may be due to the higher structural changes occurring in the blend nanocomposites with higher filler loading. This can be explained by the fact that, as the filler content is increased, higher scale structural rearrangements occur by rupturing of more and more of these filler/filler and polymer/ filler networks. For the NR/NBR nanocomposites with different 01Mt

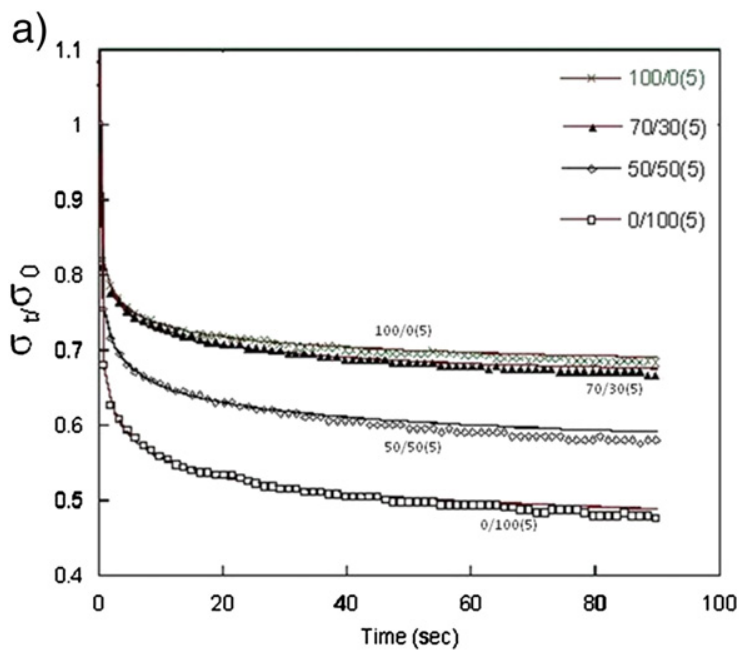

loading, this can be determined from the fitting parameter values. As the filler loading is increased, the transient stress $\sigma_{1} / \sigma_{0}$ and the characteristic relaxation time were found to be increasing, while the limiting stress value $\sigma_{\infty} / \sigma_{0}$ decreases. This can be attributed to the increased structural relaxation caused by rupturing of a higher content of polymer/filler and agglomerated filler/filler networks.

To know the relation of the stress relaxation behavior of NR/NBR/ 01Mt nanocomposite, Maxwell-Weichert model with three relaxation times was introduced and it was found that the experimental curve fitted well with the theoretical curve. The expression for stress decay according to this model is given in Eq. (3)

$\sigma t / \sigma_{0}=A \exp \left(-t / \tau_{1}\right)+B \exp \left(-t / \tau_{2}\right)+C \exp \left(-t / \tau_{3}\right)$

where $\mathrm{A}, \mathrm{B}$ and $\mathrm{C}$ are the pre-exponential factors $(\mathrm{A}+\mathrm{B}+\mathrm{C}=1)$ and $\tau_{1}, \tau_{2}, \tau_{3}$ the relaxation times, $\left(\tau_{1}<\tau_{2}<\tau_{3}\right)$. As the different relaxation times should be related to specific structures (Xia et al., 2007). The NR/NBR nanocomposite was expected to have three main relaxation processes, filler/filler disruption, polymer/filler disruption or disentanglement of network of polymer/polymer, and pullout of filler from polymer coils. The first shortest relaxation time is attributed to the breakup of filler/filler interactions, the second relaxation time is due to the disentanglement of the chains in the NR/NBR blend system, and the third longest relaxation time is from the pullout of filler particles from the polymer coils. The values of A, B, C, $\tau_{1} \tau_{2}$ and $\tau_{3}$ were calculated from the curve fitting of experimental data according to Fig. 10a \& b, and are presented in Table 5.

Fig. 10b presents the stress relaxation behavior of NR/NBR nanocomposite with $01 \mathrm{Mt}$ at different clay mineral contents. The values of A, B, C, $\tau_{1}, \tau_{2}$ and $\tau_{3}$ are presented in Table 5. It is seen from Table 5 that the value of $\tau_{1}$ increases with increase in filler loading, indicating an increase in filler/filler breakage. However, for $\tau_{2}$ and $\tau_{3}$ although it increased with OMt loading, at $5 \mathrm{phr}$ a sudden decrease in the values was observed. Since the value of $\tau_{2}$ and $\tau_{3}$ corresponds to the distanglement of polymer chains and breakage of polymer/filler interactions respectively, the decrease in value of $\tau_{2}$ and $\tau_{3}$ can be attributed to the better dispersion and interaction of 01Mt with the polymer chains. The values of the pre-exponential factors $A, B$ \& $C$ indicating the fraction of the chains undergoing relaxation also satisfy to the explanation. The clay mineral particles cannot be elongated during stretching due to high stiffness. Thus, the extension level of polymer matrix in filled NR/NBR nanocomposites was higher than that in unfilled polymer. The same trend was observed in Kohlrausch model also.

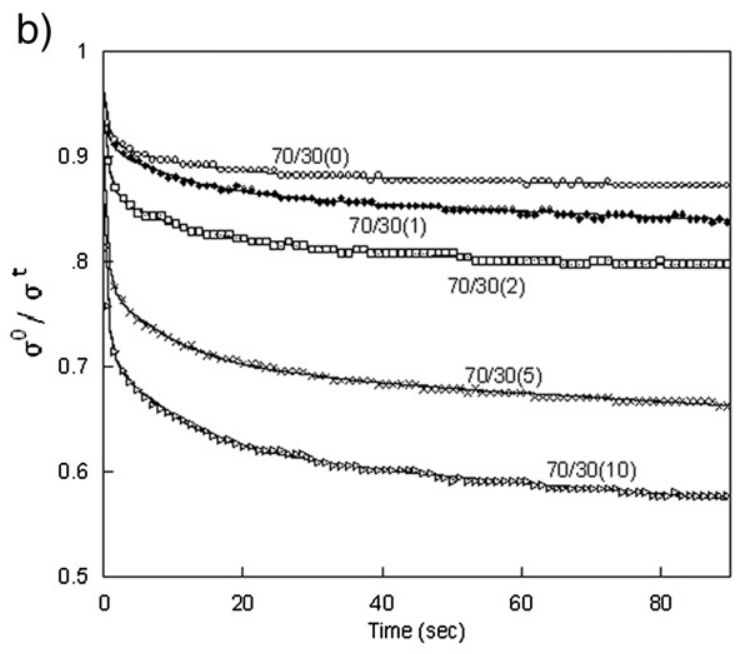

Fig. 10. Time dependence of normalized stress for a)different NR/NBR blend nanocomposites with Cloisite $10 \mathrm{~A}$ and $\mathrm{b}$ ) different C10A loaded 70/30 NR/NBR blend (curve fitting with three element Maxwell-Weichert model). 
Table 5

Curve fitting results for stress relaxation data according to Fig. 10a and b.

\begin{tabular}{|c|c|c|c|c|c|c|c|c|}
\hline Composition & $\tau 1$ & $\tau 2$ & $\tau 3$ & A & B & C & chisq & $\mathrm{R}$ \\
\hline $70 / 30(0)$ & 1099.9 & 0.5218 & 10.49 & 0.6216 & 0.1266 & 0.0966 & 0.4741 & 0.9977 \\
\hline $70 / 30(1)$ & 1705.7 & 0.5489 & 10.75 & 0.6982 & 0.1148 & 0.0803 & 0.3537 & 0.9974 \\
\hline $70 / 30(2)$ & 3524.6 & 0.8044 & 12.27 & 0.8157 & 0.0664 & 0.0521 & 0.3194 & 0.9946 \\
\hline $70 / 30(5)$ & 3121.5 & 0.4927 & 11.16 & 0.8636 & 0.0433 & 0.0506 & 0.2621 & 0.9950 \\
\hline $70 / 30(10)$ & 6684.5 & 0.7140 & 11.62 & 0.8831 & 0.0461 & 0.0320 & 0.2109 & 0.9901 \\
\hline $100 / 0(5)$ & 1718.0 & 0.5328 & 8.15 & 0.7178 & 0.1085 & 0.0786 & 0.3252 & 0.9966 \\
\hline $70 / 30(5)$ & 1641.6 & 0.5696 & 10.82 & 0.7038 & 0.1142 & 0.0791 & 0.2844 & 0.9975 \\
\hline $50 / 50(5)$ & 1162.5 & 0.8382 & 12.63 & 0.6220 & 0.1302 & 0.0882 & 0.5810 & 0.9967 \\
\hline $0 / 100(5)$ & 803.14 & 0.4465 & 8.34 & 0.5329 & 0.1822 & 0.1148 & 0.5718 & 0.9973 \\
\hline
\end{tabular}

\section{Conclusions}

The blending of NBR with NR was found to improve the physical and mechanical properties of NBR. The stress relaxation experiment was conducted in order to characterize the time-dependent reinforcement of the clay mineral reinforced NR/NBR nanocomposites. The study of the effect of filler loading showed a noticeable change in the relaxation rate. The rate of relaxation was found to decrease at lower filler loading while at higher filler loading, the rate of stress relaxation is increased. This higher relaxation rate is attributable to the decrease in polymer filler interactions. At higher loading, the increase in filler/filler interactions accelerates the stress relaxation. The nature of OMt was also found to influence the stress relaxation behavior. The 02Mt clay mineral showed lower relaxation rate compared with O2Mt because of its better interaction with the polymer matrix. Temperature also had an effect on the stress relaxation rate. Finally, it is important to add that, in order to design high performance polymer/polymer/OMt nanocomposites for dynamic applications, the OMt should have a high extent of dispersion in the matrix. The nature of modification in OMt determines the polymer/filler interactions. The experimental value of the stress relaxation curve fitted well with the theoretical value of the stretched exponential Kohlrausch equation and the three elements Maxwell-Weichert model with three relaxation times.

\section{Acknowledgments}

We thank the Department of Science \& Technology (DST)-Nano Science and Technology (Nano Mission), Delhi, India for the financial support, Jozef Stefan Institute, Department F4 Jamova Cesta 39 SI1000 Ljubljana, Slovenia for support and guidance, Ecole de mine Albi for SEM and XRD measurements and the English India clay, Thiruvananthapuram, Kerala, India for providing the nanoclay.

\section{References}

Abu-Abdeen, M., 2010. Single and double-step stress relaxation and constitutive modeling of viscoelastic behavior of swelled and un-swelled natural rubber loaded with carbon black. Mater. Des. 31, 2078-2084.
Aklonis, J.J., MacKnight, W.J., Shen, M., 1972. Introduction to Polymer Viscoelasticity. Wiley-Interscience, New York 249.

Bhagawan, S.S., Tripathy, D.K., De, S.K., 1987. Stress relaxation in short jute fiber-reinforced nitrile rubber composites. J. Appl. Polym. Sci. 33, 1623-1639. http://dx.doi.org/10.1002 app.1987.070330517.

Cao, F., Sadhan, C.J., 2007. Nanoclay-tethered shape memory polyurethane nanocomposites. Polymer 48 (13), 3790-3800.

Choi, G.D., Jo, W.H., Kim, H.G., 1996. The effect of the viscosity ratio of dispersed phase to matrix on the rheological, morphological, and mechanical properties of polymer blends containing a LCP. J. Appl. Polym. Sci. 59, 443-452. http://dx.doi.org/10.1002/ (SICI)1097-4628(19960118)59:3<443::AID-APP8>3.0.CO;2-P.

Essawy, D., El-Nashar, 2004. The use of montmorillonite as a reinforcing and compatibilizing filler for NBR/SBR rubber blend. Polym. Test. 23, 803-807.

Everaert, V., Aerts, L., 1999. Phase morphology development in immiscible PP/(PS/PPE) blends influence of the melt-viscosity ratio and blend composition. Polymer 40, 6627-6644.

Favis, B.D., Chalifoux, J.P., 1987. The effect of viscosity ratio on the morphology of polypropylene/polycarbonate blends during processing. Polym. Eng. Sci. 27, 1591-1600. http://dx.doi.org/10.1002/pen.7602721053 (Reactive Polymer Blending edited by Warren E. Baker, Chris E. Scott, G. H. Hu).

Ferry, J.D., 1970. Viscoelastic Properties of Polymers. John Wiley \& Sons, New York.

Flink, P., Stenberg, B., 1990. An indirect method which ranks the adhesion in natural rubber filled with different types of cellulose fibres by plots of $\mathrm{E}(\mathrm{t}) / \mathrm{Et}=0$ versus $\log \mathrm{t}$. $\mathrm{Br}$. Polym. J. 22, 193-199.

Freeman, G. M., Carl, J., Marshall, Jr., Lackey, W. O., Onizawa, M., 2001. Hydrous clay slurry mixture containing a silane-treated clay United States Patent 6197105, July 6th.

Fritzsche, J., Das, A., Jurk, R., Stöckelhuber, K.W., Heinrich, G., Klüppel, M., 2008. Relaxation dynamics of carboxylated nitrile rubber filled with organomodified nanoclay. Express Polym. Lett. 2, 373-381.

Fritzsche, J., Meier, J.G., Klüppel, M., 2009. Kautsch. Gummi Kunstst. 62, 319-325.

Geethamma, V.G., Pothen, L.A., Bhaskar, R., Neelakantan, N.R., Thomas, S., 2004. Tensile stress relaxation of short-coir-fiber-reinforced natural rubber composites. J. Appl. Polym. Sci. 94 (1), 96-104.

Hotta, S., Paul, D.R., 2004. Nanocomposites formed from linear low density polyethylene and organoclays. Polymer 45, 7639 .

Le, H.H., Ali, Z., Ilisch, S., Radusch, H.-J., 2010. Time-dependent reinforcement effect of nanoclay in rubber nanocomposites. J. Mater. Sci. 46, 1685-1696. http://dx.doi.org/ 10.1007/s10853-010-4986-2.

Loan, T.V., Emmanuel, P.G., 2007. Compatibilizing poly (vinylidene fluoride)/nylon-6 blends with nanoclay. Macromolecules 40 (23), 8271-8276.

Meera, A.P., Said, S., Grohens, Y., Luyt, A.S., Thomas, S., 2009. Tensile stress relaxation studies of $\mathrm{TiO}_{2}$ and nanosilica filled natural rubber composites. Ind. Eng. Chem. Res. 48, 3410-3416.

Meeting of the American Chemical Society, 1914. (American Chemical Society, Cleveland, Ohio, April 4).

Paul, D.R., Bucknall, C.B., 2000. Polymer Blends. John Wiley \& Sons, New York.

Rafailovic, M., Sokolov, J., Zhu, S., Chu, B., 2002.Compatibilizer for immiscible polymer blends, United States Patent No. 6339121, January 15.

Ray, S., Shanmugharaj, A.M., Bhowmick, A.K., 2002. A new parameter for interpretation of polymer-filler and filler-filler interactions in rubber vulcanizates. J. Mater. Sci. Lett. 21, 1097-1100. http://dx.doi.org/10.1023/A:1016506532606.

Shah, R.K., Paul, D.R., 2006. Comparison of nanocomposites prepared from sodium, zinc, and lithium ionomers of ethylene/methacrylic acid copolymers. Macromolecules 39, 3327. http://dx.doi.org/10.1021/ma0600052.

Shah, D., Maiti, P., Jiang, D.D., Batt, C.A., Giannelis, E.P., 2005a. Effect of nanoparticle mobility on toughness of polymer nanocomposites. Adv. Mater. 17, 525-528.

Shah, R.K., Hunter, D.L., Paul, D.R., 2005b. Nanocomposites from poly (ethylene-comethacrylic acid) ionomers: effect of surfactant structure on morphology and properties. Polymer 46, 2646. http://dx.doi.org/10.1016/j.polymer.01.062.

Tager, A., 1972. Physical Chemistry of Polymers. Mir Publisher, Moscow.

Varghese, S., Kuriakose, B., Thomas, S., 1994. Stress relaxation in short sisal-fiberreinforced natural rubber composites. J. Appl. Polym. Sci. 53.

Xia, H.S., Song, M., Zhang, Z.Y., Richardson, M., 2007. Microphase separation, stress relaxation, and creep behavior of polyurethane nanocomposites. J. Appl. Polym. Sci. 103, 2992. http://dx.doi.org/10.1002/app.25462.

Zheng, H., Zhang, Y., Peng, Z., Zhang, Y., 2004. Influence of clay modification on the structure and mechanical properties of EPDM/montmorillonite nanocomposites. Polym. Test. 23, 217-223. 